International Journal of Modern Physics A

(C) World Scientific Publishing Company

\title{
Jet Production in Association with Vector Bosons or Top Quarks
}

\author{
Ulla Blumenschein \\ II Physikalisches Institut, University of Goettingen, Friedrich-Hund-Platz 1 \\ Goettingen, 37077, Germany \\ ublumen@gwdg.de \\ Received 24052015 \\ Revised
}

\begin{abstract}
The LHC experiments ATLAS and CMS have measured $V+$ jets and $t \bar{t}+$ jets final states over a large energy range in data collected between 2010 and 2012 at $\sqrt{s}=7 \mathrm{TeV}$ and $\sqrt{s}=8 \mathrm{TeV}$. The results have been compared to pQCD calculations at NLO and have been used to validate novel Monte Carlo techniques.

Keywords: QCD; standard model; top

PACS numbers:
\end{abstract}

\section{Introduction}

Processes with leptons or photons in association with hadronic jets in the final state play an important role in the physics program of the LHC. They constitute in particular a typical signature of the production of top pairs and Higgs bosons. Moreover, an accumulation of these processes in certain extreme regions of phase space would be an indication of new BSM physics. Important backgrounds in the selection of Higgs and BSM processes are the associated production of gauge bosons or top quarks with jets $(t \bar{t}+$ jets, $V+$ jets $)$. Typically, the multiplicity and kinematics of jets in these events are exploited to achieve a separation from the signal, which makes the precise modelling of these quantities a high priority.

$V+$ jets and $t \bar{t}+$ jets processes also constitute powerful tests of perturbative quantum chromodynamics (pQCD), with the mass of the bosons or the top quark providing a well-defined scale. Photons and leptonic decay products of massive bosons or top quarks allow to trigger the event independently of the jet kinematics. On the theoretical side, the large progress in automated calculation of loop amplitudes ${ }^{1}$ allows for next-to-leading order (NLO) predictions with respect to a fixed order of up to five additional partons. A second path pursues the combination of tree level matrix elements ${ }^{2} \sqrt{4}$ or even NLO matrix elements ${ }^{\sqrt{5}}$ of different parton multiplicities matched to a parton shower (ME+PS, MEPS@NLO). Since the dominant graphs in basic processes contain at least one gluon in the initial state, differential or double-differential measurements of angles and momenta or masses can be used 
to constrain the gluon parton distribution function (PDF).

The signal selections start with a standard selection of inclusive $W, Z, \gamma$ or $t \bar{t}$ final states, extended by additional requirements on the presence of jets. Measured yields are typically unfolded to the particle level using regularized or iterative methods 67 and compared with fixed-order calculations and generator predictions. The latter are corrected by global k-factors to the most precise available calculation for the inclusive process. Jets are reconstructed with the anti- $k_{t}$ algorithm, ${ }^{8}$ with a distance parameter of $R=0.4$ in ATLAS and $R=0.5$ in CMS.

\section{Measurements of the $\mathrm{V}+$ jets Cross Sections}

$V+$ jets cross sections are typically measured in clean final states with electrons and muons. Although the cross section for leptonically decaying $W$ bosons is larger than for $Z$ bosons by an order of magnitude, measurements are published for both processes. Not only is it of interest to probe the impact of the small mass difference between the bosons but in particular both processes complement each other in terms of uncertainties: the $W \rightarrow \ell \nu+$ jets selection profits from the larger cross sections, whereas the $Z(\rightarrow \ell \ell)+$ jets final state can be selected with a higher purity.

Before the start of the LHC, $V+$ jets cross sections have been measured at the Tevatron in $p \bar{p}$ collisions at center-of-mass energies of $\sqrt{s}=1.96 \mathrm{TeV}, \underline{912}$ where the production is dominated by quark annihilation. For moderate transverse momentum regimes, the results confirmed the NLO calculations for $V+\geq 1$ jet and $V+\geq$ 2 jets. $\frac{13}{13}$ The data was also compared with early releases of ME+PS generators.

In contrast, $V+$ jets production in $p p$ collisions is dominated by the Compton process. The larger center-of-mass energies by the LHC enhance the QCD radiation considerably and allow to explore large jet multiplicities and large momentum ranges relevant for Higgs and BSM physics. ATLAS and CMS experiments complement each other, both in the selection of observables and of the theory predictions. Typical minimal jet transverse momenta are $p_{\mathrm{T}}^{\text {jet }} \geq 30 \mathrm{GeV}$, considerably lower than in the di-jet or multi-jet measurements.

During the first years of LHC data taking, novel NLO techniques ${ }^{11}$ resulted in a series of new fixed-order predictions for the production of massive gauge bosons with up to five jets $14 \sqrt{15}$ The LHC experiments have used the data collected in 2011 at $\sqrt{s}=7 \mathrm{TeV}$, corresponding to an integrated luminosity of $4.6-5.0 \mathrm{fb}^{-1}$ in order to probe these new predictions $\frac{16}{21} \mathrm{In}$ addition, the performance of LO and NLO ME+PS generators was studied. The results confirm the validity of the fixedorder NLO predictions for vector bosons produced in association with up to five jets performed with the new techniques. In general, ME+PS generators show a reasonable performance in modelling inclusive and differential jet cross sections. Figure 1 a shows exemplarily the $W+$ jets cross section with respect to the jet multiplicity measured by the CMS experiment ${ }^{21}$ compared with fixed-order NLO calculations and predictions from ME+PS generators. Figure $1 \mathrm{~b}$ shows the ratio of $W+$ jets and $Z+$ jets cross sections as a function of the exclusive jet multiplicity ${ }^{18}$ The ratio is 

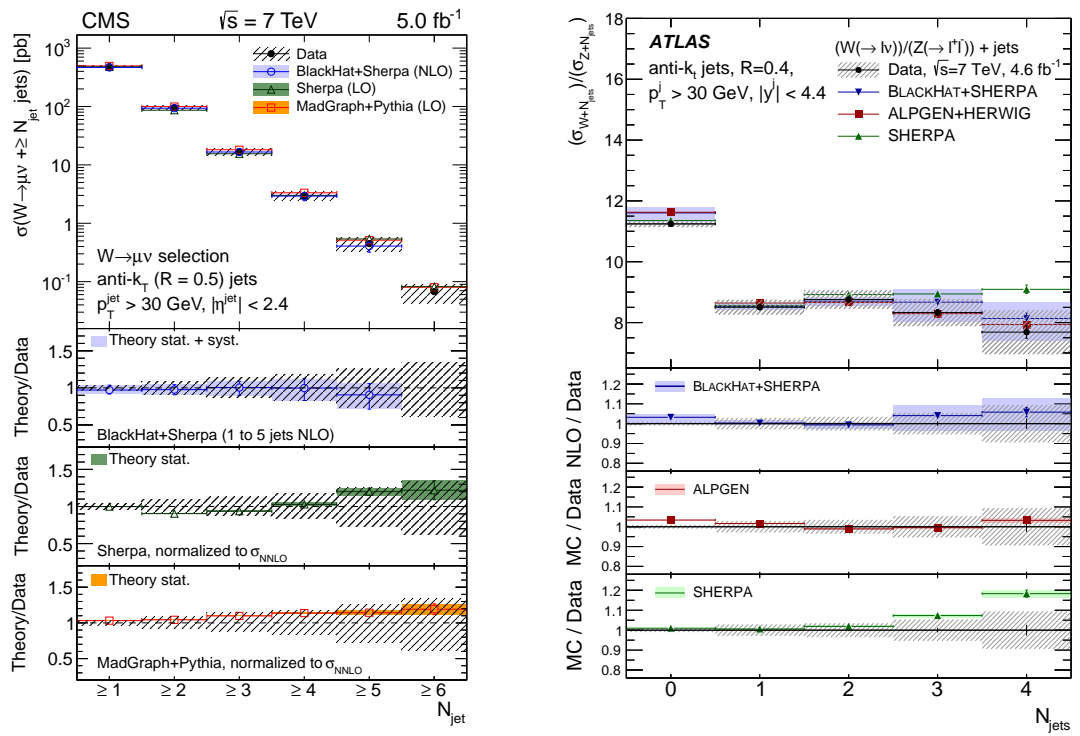

Fig. 1. (a) $W \rightarrow \mu \nu+$ jets cross section with respect to the inclusive jet multiplicity measured by the CMS experiment 21 (b) Ratio of $W+$ jets and $Z+$ jets cross sections as a function of the exclusive jet multiplicity measured by the ATLAS experiment 18 The data is compared with fixed order calculations by BLACKHAT+SHERPA and with predictions from the ME+PS generators SHERPA, MADGRAPH and ALPGEN.

well modelled both by fixed-order calculations and the generators.

The good description is preserved for exclusive jet multiplicities, even after increasing the scale difference between the leading jet and the jets which are vetoed to define the exclusive final state or after selecting events with large di-jet masses and rapidity distance $\underline{16}$

The data at center-of-mass energies of $7 \mathrm{TeV}$ and $8 \mathrm{TeV}$ allows to explore the event topology in a kinematic range up to the $\mathrm{TeV}$ scale. The list of observables includes jet transverse momenta and rapidity, angles between the final-state particles and the di-jet mass. The measurements show in general a good performance of fixedorder calculations and ME+PS generators but also reveal some deficiencies. As an example, Fig. 2 a shows the theory/data ratio of the $W+$ jets cross section measured by the ATLAS experiment as a function of the scalar transverse momentum sum $H_{\mathrm{T}}$ of all final state objects. ${ }^{[17}$ The fixed-order $W+$ jets calculation underestimates large values of $H_{\mathrm{T}}$ characterized by larger parton multiplicities. ME+PS generators prove to be better suited for modelling the $H_{\mathrm{T}}$ observable but overestimate the cross section for hard jets. The performance of SHERPA is improved by including explicit NLO matrix elements (MEPS@NLO). Figure $2 \mathrm{~b}$ shows exemplarily the $Z+\geq 2$ jets cross section normalized to the inclusive $Z$ cross section as a function of the dijet mass compared with predictions from two generators ${ }^{19}$ The distribution is well modelled at NLO by the generator POWHEG. ${ }^{22]}$ 

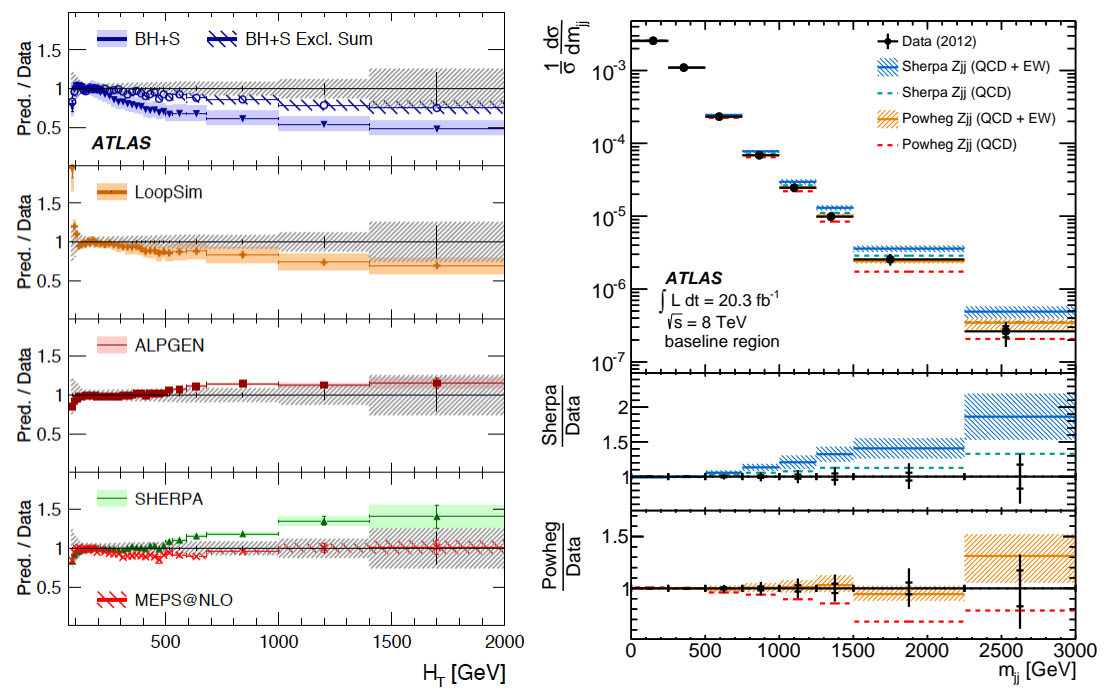

Fig. 2. (a) $W+$ jets cross section as a function of $H_{\mathrm{T}}$ in data collected with the ATLAS experiment at $\sqrt{s}=7 \mathrm{TeV}$ !17 (b) $Z+\geq 2$ jets cross section measured by the ATLAS experiment normalized to the inclusive $Z$ cross section as a function of the dijet mass $m_{j j}$ for events with $p_{\mathrm{T}}^{\text {jet } 1}>55 \mathrm{GeV}$ and $\left.p_{\mathrm{T}}^{\text {jet2 }}>45 \mathrm{GeV} 19\right]$ The data is compared with fixed order calculations by BLACKHAT+SHERPA and with predictions from the ME+PS generators SHERPA and ALPGEN and the generator POWHEG.

\section{Measurements of the Photon+Jets Cross Sections}

Isolated photons in association with jets provide an important probe of pQCD over a large energy range in a comparatively clean final state. The production of these events is dominated by the Compton process, such that they can be used in addition to the inclusive photon data to constrain the gluon PDF. In addition, $\gamma+$ jets constitutes a major background to the Higgs $\rightarrow \gamma \gamma$ process and to several BSM topologies.

The LHC experiments have measured $\gamma+$ jets cross sections in the 2010 and in the 2011 data sets at $\sqrt{s}=7 \mathrm{TeV} \sqrt{23}$ Typically, a photon isolation requirement is employed in order to enhance the purity of prompt direct photon production with respect to prompt photons from fragmentation and non-prompt photons from hadron decays in di-jet events. The published cross sections include the remaining fragmentation component. Differential cross sections are measured with respect to characteristic event quantities. In order to optimize the PDF sensitivity, double or triple differential cross sections are extracted as a function of the photon transverse energy, the photon pseudorapidity and the jet pseudorapidity. The unfolded data are compared with fixed-order NLO pQCD calculations by the JETPHOX programme ${ }^{26}$ and with predictions from ME+PS generators. Photon and jet minimum transverse momenta are typically in the range of $30-45 \mathrm{GeV}$. Dominant systematic uncertainties arise from the determination of the background from non-prompt photons. 

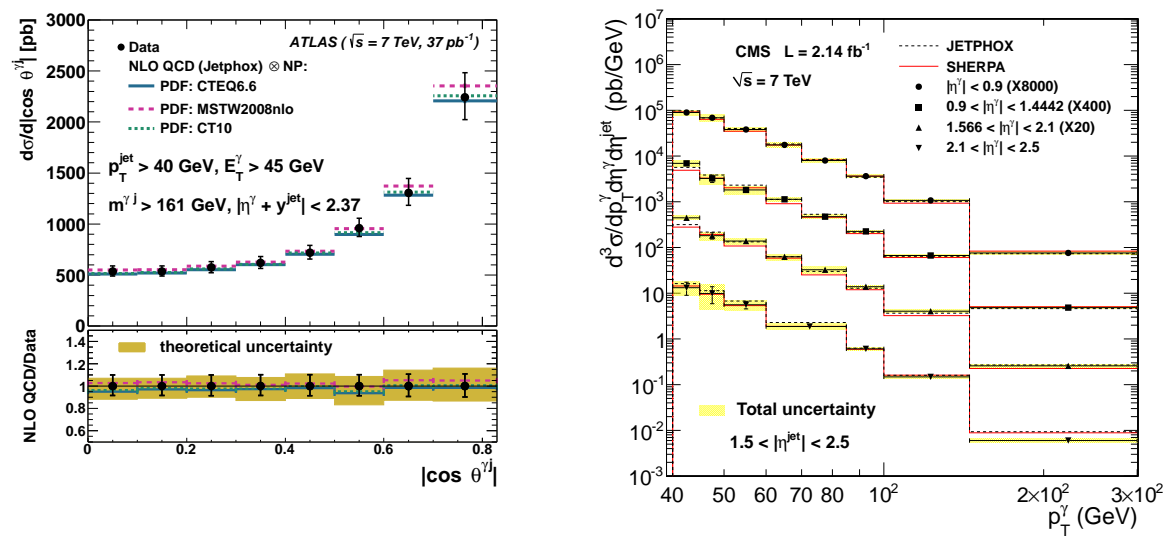

Fig. 3. (a) Differential cross section for isolated $\gamma+$ jets with respect to $\cos \theta^{*}$ measured by the ATLAS experiment 24 (b) Part of the triple-differential $\gamma+$ jets cross section measurement performed by CMS 25

As an example, Fig. 3a shows an early measurement performed by ATLAS of the scattering angle $\theta^{*}$ in the photon-jet centre-of-mass frame in a region not distorted by the kinematic preselection. The measurement confirmes the validity of the NLO pQCD calculation by JETPHOX and in particular the dominance of the Compton process in the isolated $\gamma+$ jets sample. Figure $3 \mathrm{~b}$ shows part of the triple-differential cross section measurement performed by CMS as a function of the photon transverse energy, the photon pseudorapidity and the jet pseudorapidity in the 2011 data set. The double and triple differential LHC data confirms again the good performance of the pQCD calculations and of the predictions by the LO ME+PS generator SHERPA. $\gamma+$ jets data collected at $\sqrt{s}=7 \mathrm{TeV}$ is expected to constrain the gluon PDF by up to $20 \% .27$

\section{Measurements of the $t \bar{t}+$ jets Cross Sections}

About half of the $t \bar{t}$ events produced at the LHC are accompanied by additional hard hadronic jets which do not originate from the top quark decay. These events form part of the signal selection for $t \bar{t}$ precision measurements and constitute an important background to processes with multijet final states, in particular in the Higgs and BSM sector. They also serve as an important probe of pQCD with the top quark mass providing a large well-defined scale.

ATLAS and CMS have measured $t \bar{t}+$ jets cross sections in data collected in 2011 at $\sqrt{s}=7 \mathrm{TeV}$. Both experiments select $t \bar{t}$ events in the di-lepton and in the lepton+jets final states with two resp. four jets expected from the $t \bar{t}$ decay itself. The dominant systematic uncertainty for large jet multiplicities arises from the jet energy scale. Cross sections are published for various minimum $p_{\mathrm{T}}^{\text {jet }}$ thresholds. The ATLAS collaboration measures cross sections as a function of the jet multiplicity and the jet transverse momenta. CMS publishes cross sections as a function of 

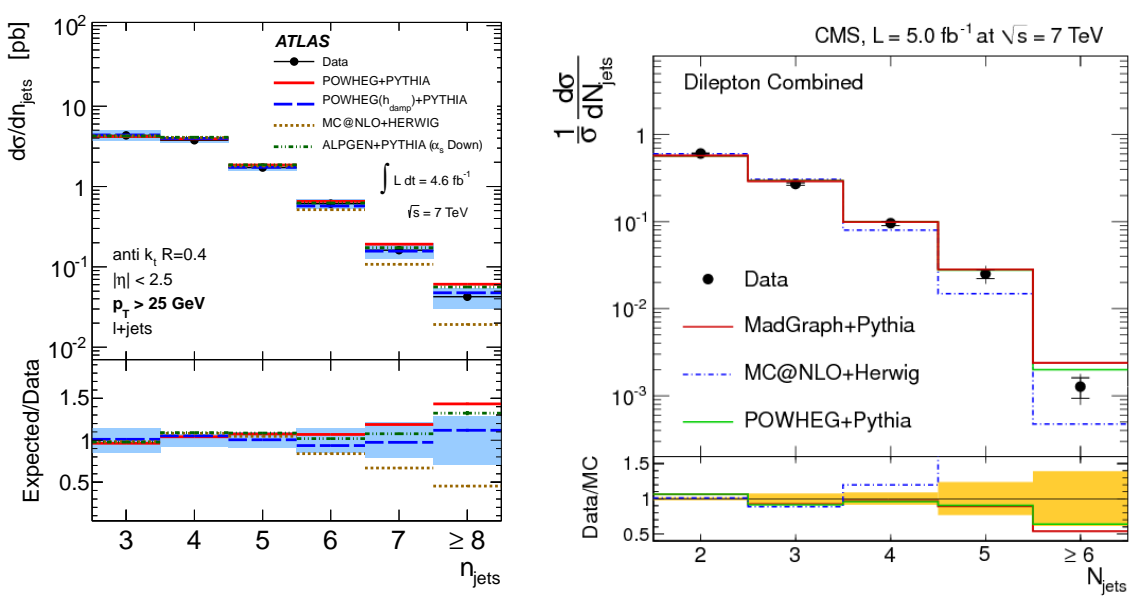

Fig. 4. (a) $t \bar{t}+$ jets cross section as a function of the jet multiplicity $\left(p_{\mathrm{T}}^{\text {jet }}>25 \mathrm{GeV}\right)$ measured by ATLAS in the lepton+jets channel ${ }^{28}$ (b) $t \bar{t}+$ jets cross section as a function of the jet multiplicity $\left(p_{\mathrm{T}}^{\mathrm{jet}}>30 \mathrm{GeV}\right)$ in the dilepton channel normalized to the inclusive $t \bar{t}$ cross section measured by the CMS experiment 29

the jet multiplicity normalized to the inclusive $t \bar{t}$ cross section to cancel part of the systematic uncertainties. Measurements are compared to predictions of ME+PS generators, where additional radiation of up to three jets is modelled by explicit matrix elements, and to NLO predictions from POWHEG and MC@NLO, where the production of more than one additional jet is modelled by the parton shower.

Figure 4 a shows exemplarily the $t \bar{t}+$ jets cross section as a function of the multiplicity of jets with $p_{\mathrm{T}}^{\text {jet }}>25 \mathrm{GeV}$ measured by the ATLAS experiment in the lepton+jets channel. ${ }^{[28}$ Figure 40 shows the corresponding normalized measurement in the dilepton channel, performed by the CMS experiment for jets with

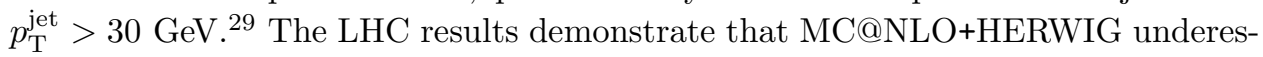
timates the radiation of more than one additional parton, while the ME+PS generators and POWHEG+PYTHIA provide a reasonable description of the additional jet activity for all $p_{\mathrm{T}}^{\text {jet }}$ thresholds.

\section{Summary and Outlook}

The LHC experiments ATLAS and CMS have measured $V+$ jets and $t \bar{t}+$ jets final states over a large energy range in data collected between 2010 and 2012 at $\sqrt{s}=7 \mathrm{TeV}$ and $\sqrt{s}=8 \mathrm{TeV}$. The results have been compared to pQCD calculations at NLO and have been used to validate novel Monte Carlo techniques. In general, a good performance of fixed-order NLO calculations and ME+PS generators is observed. Some generators show tensions in more extreme phase space regions. Double and triple-differential measurements of angles, invariant masses and transverse momenta can be used to constrain PDFs. Many measurements at $\sqrt{s}=8 \mathrm{TeV}$ are still ongoing and more results are expected in the coming year. 


\section{References}

1. C. F. Berger et al., Phys. Rev. D 78, 036003 (2008).

2. M. Mangano et al., JHEP 0307, 001 (2003).

3. T. Gleisberg et al., JHEP 0902, 007 (2009).

4. J. Allwall et al., JHEP 06 (2011) 128.

5. S. Höche et al., JHEP 1304, 027 (2013).

6. A. Hoecker, V. Kartvelishvili, Nucl. Instrum. Meth. A 372, 469 (1996).

7. G. D'Agostini, Nucl. Instrum. Meth. A 362, 487 (1995).

8. M. Cacciari, G. P. Salam and G. Soyez, 5 The anti- $k_{t}$ jet clustering algorithm, JHEP 0804, 063 (2008).

9. CDF Collab. (T. Aaltonen et al.), Phys. Rev. Lett. 100, 102001 (2008).

10. CDF Collab. (T. Aaltonen et al.), Phys. Rev. D 77, 011108 (2008).

11. D0 Collab. (V. M. Abazov et al.), Phys. Lett., B 669, 278 (2008).

12. D0 Collab. (V. M. Abazov et al.), Phys. Lett. B 682, 370 (2010).

13. J. Campbell and R. K. Ellis, Phys.Rev. D65, 113007 (2002).

14. C. F. Berger et al., Phys. Rev. D 80, 074036 (2009); C. F. Berger et al., Phys. Rev. Lett. 106, 092001 (2011).

15. C. F. Berger et al., Phys. Rev. D 82, 074002 (2010); H. Ita et al., Phys. Rev. D 85 (2012) 031501; Z. Bern et al., Phys. Rev. D 88, 014025 (2013).

16. ATLAS Collab., (G. Aad et al.), JHEP 07, 032 (2013).

17. ATLAS Collab., (G. Aad et al.), Eur.Phys.J. C 75, 75:82 (2015).

18. ATLAS Collab., (G. Aad et al.), Eur.Phys.J. C 74, 3168 (2014).

19. ATLAS Collab., (G. Aad et al.), JHEP 04, 031 (2014).

20. CMS Collab. (S. Chatrchyan et al.), Phys. Rev. D 91, 052008 (2015); CMS Collab. (S. Chatrchyan et al.), Phys. Lett. B 722, 238 (2013); CMS Collab. (S. Chatrchyan et al.), Phys. Rev. D 88, 112009 (2013).

21. CMS Collab. (S. Chatrchyan et al.), Phys. Lett. B 741, 12 (2015).

22. S. Alioli et al., JHEP 1006, 043 (2010).

23. ATlAS Collab., (G. Aad et al.), Phys. Rev. D 85, 092014 (2012).

24. ATLAS Collab., (G. Aad et al.), Nucl. Phys. B 875, 483-535 (2013).

25. CMS Collab. (S. Chatrchyan et al.), JHEP 06, 009 (2014).

26. S. Catani et al., JHEP 05, 028 (2002).

27. L. Carminati et al., Europhys. Lett. 101, 61002 (2013).

28. ATLAS Collab., (G. Aad et al.), JHEP 01, 020 (2015).

29. CMS Collab. (S. Chatrchyan et al.), Eur.Phys.J. C 74, 3014 (2014). 